\title{
Towards Design Principles for Pharmacist-Patient Health Information Systems
}

\author{
Dirk Volland $^{1}$, Klaus Korak ${ }^{2}$, David Brückner ${ }^{2}$, and Tobias Kowatsch ${ }^{1}$ \\ ${ }^{1}$ University of St. Gallen, Dufourstrasse 40a, 9000 St. Gallen, Switzerland \\ \{dirk.volland, tobias.kowatsch\}@unisg.ch \\ ${ }^{2}$ Konsortium Pilot AlphaStreams, Weinbergstr. 56/58, 8092 Zürich, Switzerland \\ $\{\mathrm{kk}, \mathrm{db}\}$ @alphastreams. com
}

\begin{abstract}
A significant drawback of communications between patients and health professionals is their restriction to face-to-face encounters within healthcare institutions. This limits the support health professionals can provide to ensure patient adherence, which is a significant contributor to therapeutic outcome and overall healthcare expenses. Pharmacist-patient health information systems (PPHIS) have the potential to address existing non-adherence behaviors by enabling pharmacist-patient communication over the time of therapy. Due to the lack of prior research, design principles for PPHIS are derived from the information-, motivation-, and strategy model [4] and feedback from pharmacists in 21 Swiss pharmacies. To demonstrate the feasibility of the design principles, we implement and preliminarily evaluate a PPHIS.
\end{abstract}

Keywords: Pharmacist-Patient Health Information System, Design Principles, Mobile Information System, Prototype, Adherence, Communication.

\section{Introduction}

One of the key challenges in healthcare is to continuously balance access to and quality of care for individuals with associated costs for society. While most attempts focus on institutional efficiency and therapeutic progress, the patient herself has been described as healthcare's greatest untapped resource [6]. Patients however, are never simply isolated individuals, but are characterized by specific interactions and relationships with institutionalized health professionals. This provides an opportunity for healthcare information systems (HIS).

For patients and health professionals alike, relationships are still heavily influenced by a fundamental asymmetry of being on "the inside" or "the outside" of healthcare institutions such as pharmacies, physicians' practices, and hospitals. For the patient, leaving those institutions means leaving well-structured pathways where guidance and support is directly provided by health professionals. The absence of guidance outside institutions often has detrimental effects on patient adherence, and thus on therapy outcomes and associated costs. Adherence and non-adherence describe patients' behavior outside healthcare institutions, to either follow recommended guidance and treatment advice from health professionals, or not [4]. The underlying reasons for 
non-adherence are manifold. Targeting single factors often does not lead to a desired improvement [3]. Due to a pharmacy's unique position within the healthcare system, providing direct and cost-efficient access to medical experts, products, and services, as well as often being the last or sole care provider in personal contact with patients beginning their therapy, pharmacist-patient health information systems (PPHIS) have the potential to address non-adherence by allowing pharmacists to guide patients during therapy. However, to date no design principles exist that describe the generic design of such systems given the existing variety of medical diagnoses and therapies.

In response to these facts, the current work addresses the question of how PPHIS can overcome the spatial and temporal boundaries between patients and health professionals enabling a continuous and structured communication to improve patient adherence. To address this question, overall research follows an iterative design science methodology $[5,10]$. That is, several build and evaluate loops are planned during the design of principles for PPHIS that support patient adherence. In particular, the current work first justifies its relevance by a literature review in Section 2. In Section 3, design principles for PPHIS are developed that support the communication between patients and health professionals with the overall goal to increase patient adherence in everyday situations outside healthcare institutions. These principles are informed by the information-, motivation-, and strategy (IMS) model [4] as a kernel theory for improving patient adherence and, from a practitioner's point of view, by feedback from pharmacists in 21 Swiss pharmacies. The PPHIS is prototypically implemented according to these principles and preliminary evaluated as described in Section 4. This work-in-progress concludes with a summary and an outlook on future research.

\section{Literature Review}

In order to identify existing work with a focus on design principles for HIS in general and PPHIS in particular, a search strategy of prior work was adopted [2, 11]. The list of journals of prior reviews was retained and enriched by articles from the DESRIST conferences and the journal Health Systems. The following three publications are the final result of an iterative search and subsequent rigorous analysis of publications between 2002 and 2013. ${ }^{1}$

The authors of the first article [7] propose an approach for designing an ubiquitous IS to reduce adverse drug events of patients by improving communication between health professionals and patients. Using the health promotion model as a kernel theory they derive five design principles. They specifically point to the difficulty of integrating the health promotion model into the design model and call for a deeper integration of kernel theories into the design process. The second publication describes the design and evaluation of a smart medication management system for improving medication adherence [14]. This paper also builds on the health promotion model. In the third paper, a framework is described that is designed to support the requirements of patient

1 Exact search procedure, queries, and the resulting list of publications and applied criteria can be retrieved from https://dl.dropbox.com/u/2264855/2013-02-5Literature-Review.xlsx 
monitoring with a focus on emergency messages [13]. However, design principles for HIS with the goal to address adherence and communication situations when patients have left healthcare institutions were not found.

\section{Design Principles for PPHIS}

In the current work, the design principles for PPHIS are primarily derived from the IMS model as the kernel theory with a focus on health professional-patient communication and adherence. This model specifically focuses on the practical application of health behavior change. It builds upon multiple classic approaches to health behavior and incorporates elements from the entire range of healthcare professional-patient communication [4]. The authors offer three broad categories of achievement including goals, actions, and accomplishments to guide health professionals and patients towards adherence: (1) Information, (2) Motivation, and (3) Strategy. It is the task of health professionals to tailor the given categories to individual patients.

In addition to the IMS model, and given the importance of aligning human, organizational, and technological factors [15], the requirements derived from the IMS model are enriched and merged with findings from 21 semi-structured interviews in Swiss pharmacies, conducted in the last quarter of 2012, and 19 responses to a standardized questionnaires completed subsequent to the personal interviews. One to four pharmacists participated in these interviews. Personal interviews addressed the current situation of pharmacies, pharmacist-patient communication, the existence of after-care operations, methods to control, support, or identify adherence, and the requirements for a PPHIS to support patients subsequent to a pharmacy visit. The questionnaire aimed to quantify different themes surrounding topics of adherence and patient support by the pharmacist. ${ }^{2}$ For the analysis of the interviews, themes were extracted by using conceptual and relationship codes [1]. Finally, as interactions between health professionals and patients are extended beyond sole face-to-face communications, emerging requirements of temporal and spatial dimensions are of central importance for the development of the design principles [8, 9]. In the following, five design principles are developed based on the IMS model and feedback from 21 pharmacies.

First, the IMS model originally targets face-to-face encounters, which are temporally and spatially limited to the pharmacy visit. Mobile computing has dramatically changed work practices and communication in a variety of domains, freeing users from the confines of fixed locations and time. Smartphones hold the potential to structure and influence the spatial and temporal arrangements of both actors and their actions beyond existing boundaries. The perspective of temporal structures that actors engage in as part of their everyday routines has been introduced by [9]. Examples of temporal structures are weekly meetings or project deadlines that can be "used by people to give rhythm and form to their everyday work practices" (ibid., p. 685). Likewise, these temporal structures can serve as powerful templates that may help to structure patients' therapies. These theoretical implications were also identified as

2 The interview guidelines and questionnaires can be requested from the authors. 
the most prominent theme from the interview analysis. Pharmacists perceive it as a significant limitation that once the patient has stepped out, nothing is known about her progress and no further support can be provided. All participating pharmacists of the 21 pharmacies agreed that today no structured methods to follow up with patients exist. Six pharmacists ask patients in some cases to return to the pharmacy after a certain period of time (e.g. after 5 days) or meetings are set in advance. Telephone or e-mail support is possible as reported by four pharmacists, but is only applied in complex cases. Methods to check or control patients' adherence are limited to the face-toface encounter, and in rare and complex cases to dosing and supporting systems. On average $5 \%$ of patients contact the pharmacy because of questions during therapy using phone or e-mail. By contrast, approximately $11 \%$ of patients return to the pharmacy during therapy. Based on the discussion of the IMS model and the findings from the pharmacies above, the first design principle is formulated as follows:

DP1: The PPHIS should allow a pharmacist to follow up with a patient and communicate with a patient during the time of therapy.

Second, the information construct of the IMS model highlights the importance of patients' knowledge and how information, effectively communicated, can influence adherence. When patients understand, interpret, and remember health information correctly, they are much more likely to be adherent. However, existing research shows that even when the right information is provided during the encounter, a majority of patients fails to recall it later [4]. Accordingly, the second theme identified in the interviews was the perceived need of patients (as seen by the pharmacists) to be supported over the time of therapy and the willingness and perceived competency of the pharmacists to provide that support. Asked about the number of questions pharmacists think their patients have after visiting the pharmacy, 14 pharmacists responded 1-2 and 5 pharmacists responded 3-4. Asked about the share of patients, who are able to follow their therapy as recommended, opinions of pharmacists diverged, ranging from $6 \%$ to $95 \%$, with an average of $54 \%$. Pharmacists strongly agree that continuous information about the therapeutical progress can improve the quality of therapy. A one-sample t-test was applied to check if the calculated average differs from the scale's medium of 3 (Mean $=4.47, \mathrm{SD}=.70, \mathrm{p}<.001$ ) on a 5-item Likert scale from "strongly disagree" to "strongly agree". Following up with patients during their therapy could significantly improve therapy outcomes (Mean $=4.52, \mathrm{SD}=.70$, $\mathrm{p}<.001)$. At the same time, pharmacists acknowledge that they currently lack information about patients' therapy progress (Mean $=2.84, \mathrm{SD}=.96, \mathrm{p}>.25$ ). However, providing information and receiving information is mutually dependent. Based on this discussion, the second design principle is stated:

DP2: The PPHIS should allow a pharmacist to provide health-information to a patient during the time of therapy and adjust and enrich that information depending on feedback from a patient.

Third, the motivation component of the IMS model refers to situations when patients only follow treatment recommendations they believe in. Consequently, it is important that health professionals help patients to trust the efficacy of treatment recommenda- 
tions. Pharmacists need the ability to follow up with patients in case therapy does not progress as intended, the patients' confidence in treatment is low, or when the patient believes that she is not capable to adhere to the recommendations (as described above pharmacists estimate that only $54 \%$ of patients follow these recommendations). Based on the above, the third design principle is stated:

DP3: The PPHIS should allow a pharmacist to motivate a patient e.g. by promoting the perception of efficacy during the time of therapy and adjust and enrich that motivation depending on feedback from a patient.

Fourth, the strategy component of the IMS model refers to situations where concrete barriers exist that prevent patients from following treatment recommendations. Hence, health professionals need to support patients in finding a workable strategy and provide concrete assistance [4]. This can include medication reminders, but also the structured informational and motivational support over time. Pharmacists strongly believe that they can increase patient adherence in everyday practices (Mean $=4.42$, $\mathrm{SD}=0.61, \mathrm{p}<.001)$. Based on that, the fourth design principle is formulated:

DP4: The PPHIS should allow a pharmacist to provide strategic support to a patient during the time of therapy and adjust and enrich that strategy depending on feedback from a patient.

Finally, because of the heterogeneity of informational, motivational, and strategic support needed by patients, it is essential that health professionals can configure follow-up content (DP2-4) and its temporal dimension (DP1) in the situation of face-toface encounters. This corresponds to the third theme that emerged from the interviews regarding the need for seamless integration into existing pharmacists' workflows and the need for a low-investment solution. This is due to the limitations in personnel as well as workplace resources. So, on the basis of the given situation, pharmacists need the ability to situatively choose from a set of informational, motivational, and strategic elements and instantiate them at the point of patient contact. Depending on the configuration, the patient then receives adherence-supporting elements over the chosen follow-up window. Based on the above, the last design principle is stated:

DP5: At the time of the face-to-face encounter with a patient in the pharmacy, the PPHIS should allow a pharmacist to choose from a given set of informational, motivational, and strategic elements and instantiate them for the given situation over a specified time pattern.

While DP2, DP3, and DP4 are design principles concerning the content of pharmacist-patient communication, DP1 and DP5 relate to the process of communication. During face-to-face encounters of pharmacist and patient, informational, motivational, and strategic elements are instantiated over a chosen period of time. When leaving the pharmacy, the patient automatically receives these elements following a predefined time pattern as configured by the pharmacist. These elements can inform the patient about her therapy in form of, for example, text or video. They can motivate the patient to ask questions or to document her therapy progress. They can provide strategic sup- 
port, for example, by reminding the patient to take certain actions. Overall, it is a semi-automated process where pharmacists get involved depending on the actions of the patient, for example, when she asks a question or a photo is sent that documents the current state of therapy. The proposed design principles for PPHIS are flexible in that the respective elements are instantiated at the time of face-to-face encounters. They are dynamic in that the content itself (DP2-4), but also the structuring over time (DP1) and the instantiation (DP5) can be continuously improved, based on data generated through pharmacist-patient communication.

\section{Application of the Design Principles}

To demonstrate the feasibility and utility of the design principles, we implemented and preliminarily evaluated a PPHIS consisting of (1) a tangible code-element in form of a simple postcard, (2) a communication and monitoring interface for pharmacists tailored to a tablet-PC, and (3) a communication interface for patients in the form of a smartphone application. ${ }^{3}$ Besides the ability to provide information to the patient and question and answer communication, several types of feedback are implemented that can be configured for a specific time pattern by the pharmacist such as (1) making a photo e.g. of affected skin, (2) reporting general therapy progress on a scale from "significantly worse" to "significantly better", or (3) reporting simple medical parameters such as blood pressure or pulse. Currently, the code-element (Fig. 1a) consists of eight digits in the format $\mathrm{xxxx}-\mathrm{yy}-\mathrm{zz}$ that encapsulate a unique pharmacy and customer ID ( $\mathrm{xxxx})$, the structure of the content ( $\mathrm{yy})$, and the duration $(\mathrm{zz})$ of the follow-up with a particular patient (DP1 and DP5). The handover of the code does not interrupt existing workflows and bridges the offline encounter in the pharmacy with the online interaction using the PPHIS. Several configurations of content and structure are currently predefined (e.g. the patient receives the invitation to ask questions on the second day, is asked about general progress on the third day, and is asked to provide a photo on the fifth day). Flexibility of code structure and code generation will be further improved in future prototypes.

When the patient first downloads the patient app, she is asked for the code and after entering it, she receives messages and notifications in a twitter-like main screen
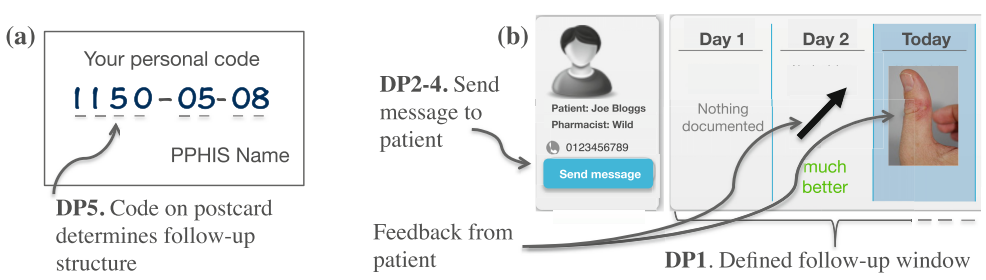

Fig. 1. Tangible code element (a) and particular patient record in iPad pharmacist app (b)

3 The primary target platforms were iPhone and iPad. However, the implementation was done in Titanium (http://appcelerator.com), consisting of an SDK that provides the necessary tools, compilers, and APIs to build for different target platforms. 


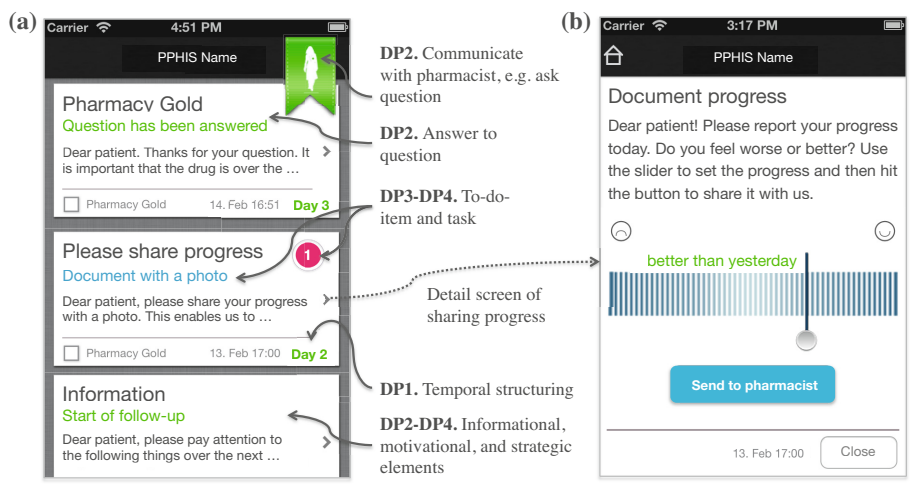

Fig. 2. Main screen (a) and screen of progress documentation (b) of iPhone patient app

(Fig. 2a). This process is automated over the specified duration and depends solely on the entered code. Informational elements that come into the stream provide healthrelated information, but other items require manual interactions such as when the patient is asked for her progress or if she has any questions related to her therapy (DP2-DP4). Further interaction or more detailed information is accessible when clicking on the messages in the main screen, for example, a screen to provide therapy progress (Fig. 2b). Only then, the pharmacist is notified in the pharmacist app and can view and respond to the feedback (Fig. 1b), or answer a question.

The PPHIS is currently pre-evaluated with 21 pharmacies. In each pharmacy, one pharmacist takes the role of the pharmacist while the other employees take patients' roles. So far, the implemented system has been tested by 21 pharmacists and more than 100 "internal patients". Log file analysis shows that the system has been extensively used (555 messages, 236 instantiations of follow-ups, and 183 documentations). Overall, qualitative feedback so far is positive, and pharmacists say that the PPHIS is easy and intuitive to use. The concept is seen as promising and pharmacies are eager to go live with their customers.

\section{$5 \quad$ Conclusions and Further Research}

In this work-in progress paper, we address the lack of prior research on design principles for PPHIS that enable pharmacist-patient communication beyond current faceto-face encounters to support patients' adherence. Accordingly, design principles for PPHIS are developed based on the IMS model and feedback from 21 pharmacies. They were finally applied during the design of a PPHIS that was pre-evaluated, too.

After the pre-evaluation of the PPHIS prototype within the pharmacies, qualitative feedback from all pharmacists will be collected once more to iterate again through the design cycle, and to proceed by testing the PPHIS including patients of participating pharmacies. To evaluate how the implemented PPHIS affects patients' adherence, a five-item self-report scale will be used as suggested in [4]. In addition to the qualitative feedback, valuable insights by analyzing PPHIS' log files and by "listening into" 
pharmacist-patient communication [12] are expected. The sample of 21 pharmacies with 50 to 500 patient encounters per day allows for a broad validation of the principles in different settings. Theoretical contributions for designing PPHIS are expected from analyzing usage patterns and communication contents by uncovering how patients can be optimally guided subsequent to a pharmacy visit. Practical contributions are expected regarding the feasibility of PPHIS in addressing population's adherence issues and thus reducing overall healthcare expenses. Finally, PPHIS might be a valuable tool for pharmacies enabling them to extend the range, value, and accessibility of their service portfolio by overcoming current spatial and temporal limitations.

\section{References}

1. Bradley, E.H., Curry, L.A., Devers, K.J.: Qualitative Data Analysis for Health Services Research: Developing Taxonomy, Themes, and Theory. Health Services Research 42(4), 1758-1772 (2007)

2. Chiasson, M.W., Davidson, E.: Pushing the contextual envelope: developing and diffusing IS theory for health information systems research. Information \& Organization 14(3), 155-188 (2004)

3. DiMatteo, M.R., Giordani, P.J., Lepper, H.S., Croghan, T.W.: Patient adherence and medical treatment outcomes: a meta-analysis. Medical Care 40(9), 794-811 (2002)

4. DiMatteo, M.R., Haskard-Zolnierek, K.B., Martin, L.R.: Improving patient adherence: a three-factor model to guide practice. Health Psychology Review 6(1), 74-91 (2012)

5. Gregor, S., Jones, D.: The anatomy of a design theory. Journal of the Association for Information Systems 8(5), 312-335 (2007)

6. Kemper, D.W., Mettler, M.: Information Therapy: Prescribed Information as a Reimbursable Medical Service (2002)

7. Maass, W., Varshney, U.: Design and evaluation of Ubiquitous Information Systems and use in healthcare. Decision Support Systems 54(1), 597-609 (2012)

8. Martins, H.M.G.: Critical Concepts in M-Health Technology Development: Time, Space, and Mobility. In: Telemedicine and E-Health Services, Policies, and Applications, pp. 140-150 (2012)

9. Orlikowski, W.J., Yates, J.: It's about time: Temporal Structuring in Organizations. Organization Science 13(6), 684-700 (2002)

10. Peffers, K., Tuunanen, T., Rothenberger, M., Chatterjee, S.: A design science research methodology for information systems research. Information Systems 24(3), 45-77 (2007)

11. Romanow, D., Cho, S., Straub, D.: Riding the Wave: Past Trends and Future Directions for Health IT Research. MIS Quarterly 36(3), iii-A18 (2012)

12. Urban, G., Hauser, J.: "Listening In" to Find and Explore New Combinations of Customer Needs. Journal of Marketing 68(2), 72-87 (2004)

13. Varshney, U.: A framework for supporting emergency messages in wireless patient monitoring. Decision Support Systems 45, 981-996 (2008)

14. Varshney, U.: Smart medication management system and multiple interventions for medication adherence. Decision Support Systems (2012)

15. Yusof, M.M., Kuljis, J., Papazafeiropoulou, A., Stergioulas, L.K.: An evaluation framework for Health Information Systems: human, organization and technology-fit factors (HOT-fit). International Journal of Medical Informatics 77(6), 386-398 (2008) 